\title{
Observations on Mohr-Coulomb Plasticity under Plane Strain
}

\author{
W. M. Coombs ${ }^{1}$; R. S. Crouch ${ }^{2}$; and C. E. Heaney ${ }^{3}$
}

\begin{abstract}
Linear elastic-perfect plasticity using the Mohr-Coulomb yield surface is one of the most widely used pressure-sensitive constitutive models in engineering practice. In the area of geotechnical engineering, a number of problems, such as cavity expansion, embankment stability, and footing bearing capacity, can be examined using this model together with the simplifying assumption of plane strain. This paper clarifies the situation regarding the direction of the intermediate principal stress in such an analysis and reveals a unique relationship between hydrostatic pressure and the principal stress ratio for Mohr-Coulomb and Tresca perfect plasticity under those plane-strain conditions. The rational relationship and direction of the intermediate principal stress are illustrated through both material-point and finite-element simulations. The latter involves the analysis of a rigid strip footing bearing onto a weightless soil and the finite-deformation expansion of a cylindrical cavity. DOI: 10.1061/(ASCE)EM.1943-7889.0000568. @ 2013 American Society of Civil Engineers.
\end{abstract}

CE Database subject headings: Stress; Stain; Elastoplasticity; Geomaterials; Constitutive models.

Author keywords: Intermediate principal stress; Mohr-Coulomb; Tresca; Elastoplasticity; plane-strain analysis; Geomaterials.

\section{Introduction}

The compressive strength and inelastic deformation of particulate materials, such as soils, fractured rocks, grains, and powders, are dependent on the effective hydrostatic pressure. This behavior is characteristic of media where the mechanics are dominated by frictional forces. The linear elastic-perfectly plastic Mohr-Coulomb (M-C) model is one of the most widely used pressure-sensitive constitutive models, which can capture this behavior in an idealized way. Although originally expressed in terms of the major and minor principal stresses, the $\mathrm{M}-\mathrm{C}$ criterion was recast in terms of all three principal dresses by Shield (1955). With zero friction, the M-C model reduces to the pressure-invariant Tresca formulation as a special case. That model has been extensively used when analyzing the elastoplastic behavior of metals [see, for example, Ewing and Griffiths (1971) and Griffiths and Owen (1971)].

A number of common geotechnical problems, such as footing displacement, embankment stability, and cavity expansion, lend themselves to two-dimensional plane-strain analysis. Such analyses can provide a useful approximation of the structural behavior while requiring only modest computational expenditure (when compared with three-dimensional analyses).

This paper presents the rational relationship between the relative magnitude of the intermediate principal stress $(b$, subsequently defined) and the hydrostatic pressure $(\xi)$ for the M-C and Tresca constitutive models under plane-strain conditions. This relationship

\footnotetext{
${ }^{1}$ Lecturer, School of Engineering and Computing Sciences, Durham Univ., Durham DH1 3LE, U.K. (corresponding author). E-mail: w.m .coombs@durham.ac.uk

${ }^{2}$ Dean, School of Engineering, Mathematics and Computer Science, City Univ. London, London EC1V OHB, U.K.

${ }^{3}$ Marie Curie Research Fellow, Plaxis BV, Delftechpark 53, 2628 XJ, Delft, Netherlands.

Note. This manuscript was submitted on May 1, 2012; approved on November 9, 2012; published online on November 12, 2012. Discussion period open until February 1, 2014; separate discussions must be submitted for individual papers. This technical note is part of the Journal of Engineering Mechanics, Vol. 139, No. 9, September 1, 2013. (ASCE, ISSN 0733-9399/2013/9-1218-1228/\$25.00.
}

expresses the principal stress locus that elastoplastic states are required to follow over the M-C yield surface.

Isotropic constitutive formulations (such as, the $\mathrm{M}-\mathrm{C}$ and Tresca models) allow the relationships to be described using principal stress and strain quantities, providing a clear geometric interpretation of the material state. All of the findings are presented using principal stresses and strains with a tension positive notation. The principal stress ratio is defined here as

$$
b=\frac{\sigma_{1}-\sigma_{2}}{\sigma_{1}-\sigma_{3}} \quad \in[0,1]
$$

where $\sigma_{1}$ and $\sigma_{3}$ are the major (most tensile) and minor (most compressive) principal stresses, respectively, such that $\sigma_{1} \geq \sigma_{2} \geq \sigma_{3}$. The hydrostatic stress is defined as $\xi=\operatorname{tr}([\sigma]) / \sqrt{3}=\left(\sigma_{1}+\sigma_{2}+\sigma_{3}\right) / \sqrt{3}$, where $\operatorname{tr}([\cdot])$ denotes the trace of $[\cdot] . \xi$ is not the mean stress, it corresponds to the distance along the hydrostatic axis from the origin in Haigh-Westergaard stress space. The principal stress ratio is related to the Lode angle, $\theta$, through

$$
\begin{aligned}
& b=\frac{1+\sqrt{3} \tan (\theta)}{2}, \quad \text { where } \\
& \theta=\frac{1}{3} \arcsin \left(\frac{-3 \sqrt{3}}{2} \frac{J_{3}}{J_{2}^{3 / 2}}\right) \quad \in[-\pi / 6, \pi / 6]
\end{aligned}
$$

The deviatoric stress invariants are given by $J_{2}=\operatorname{tr}\left([s]^{2}\right) / 2$ and $J_{3}=\operatorname{tr}\left([s]^{3}\right) / 3$, where the traceless deviatoric stress matrix $[s]$ $=[\sigma]-\xi[I] / \sqrt{3}$, and $[I]$ is the third-order identity matrix.

The layout of the paper is as follows. Initially, the M-C constitutive relationships are presented, including the isotropic linear stress-elastic strain law, yield criterion, and plastic flow direction. The next section restricts the $\mathrm{M}-\mathrm{C}$ constitutive model to the case of plane-strain analysis and derives the relationship between the hydrostatic stress, $\xi$, and the principal stress ratio, $b$. The limiting cases of triaxial compression $\left(b=0 ; \sigma_{2}=\sigma_{1}\right)$ and extension $(b=0$; $\sigma_{2}=\sigma_{3}$ ) are also considered. The simplification of the M-C $\xi$ versus $b$ relationship for the Tresca constitutive model is given, and the rational relationship is extended to account for inelastic straining in the out-of-plane direction induced by the corners present in the 
yield envelopes. After this, a simple material point investigation is used to investigate the assumption that the out-of-plane stress is indeed the intermediate principal stress. Three finite-element investigations using the $\mathrm{M}-\mathrm{C}$ model are then presented: (1) a simple two-element simulation, (2) an analysis of a rigid strip footing bearing onto a weightless soil, and (3) a finite-deformation cavity expansion simulation. These simulations provide numerical verification of the $\xi-b$ relationship for the $\mathrm{M}-\mathrm{C}$ model. Conclusions are drawn in the final section.

\section{Mohr-Coulomb Constitutive Formulation}

The constitutive laws for (and the algorithmic treatment of) the isotropic linear elastic-perfectly plastic M-C model are widely available in the literature [for example, see the papers by Clausen et al. (2006, 2007) and references cited therein]. Here, to aid clarity, the basic equations required in the later sections are reviewed briefly.

\section{Linear Isotropic Elasticity}

The following is the isotropic linear elastic stiffness matrix

$$
\left[D^{e}\right]=\frac{E}{(1+\nu)(1-2 \nu)}[(1-2 \nu)[I]+\nu[1]]
$$

which provides the relationship between the vectors containing the principal Cauchy stresses, $\{\sigma\}$, and the principal elastic strains, $\left\{\varepsilon^{\mathrm{e}}\right\}$

$$
\{\sigma\}=\left[D^{e}\right]\left\{\varepsilon^{e}\right\}
$$

In Eq. (3), $E$ is Young's modulus, $\nu$ is Poisson's ratio, and [1] is the third-order matrix populated with ones. The total strain vector is split into elastic (recoverable) and inelastic (irrecoverable) components as follows: $\{\varepsilon\}=\left\{\varepsilon^{e}\right\}+\left\{\varepsilon^{p}\right\}$.

\section{Inelasticity}

The M-C criterion assumes that plastic frictional sliding will occur once the minor principal stress, $\sigma_{3}$, falls below some proportion of the major principal stress, $\sigma_{1}$. This can be defined using the following yield function

$$
\begin{aligned}
& f=k \sigma_{1}-\sigma_{3}-\sigma_{c}=0, \quad \text { where } \\
& k=\frac{1+\sin (\phi)}{1-\sin (\phi)} \quad \text { and } \quad \sigma_{c}=2 c \sqrt{k}
\end{aligned}
$$

where $\phi=$ internal friction angle, $c=$ cohesion, and $\sigma_{c}$ defines the uniaxial compressive yield strength. The M-C yield surface is shown in Fig. 1 using (1) a deviatoric section viewed down the hydrostatic axis and (2) an isometric view of the principal stress space.

The nonassociated plastic flow direction is given by

$$
\left\{g_{, \sigma}\right\}=\left\{\begin{array}{lll}
k_{g} & 0 & -1
\end{array}\right\}^{T}, \quad \text { where } k_{g}=\frac{1+\sin \left(\phi_{g}\right)}{1-\sin \left(\phi_{g}\right)}
$$

and $\phi_{g} \in[0, \phi]=$ plastic dilation angle, such that the rate of inelastic straining is given by

$$
\left\{\dot{\varepsilon}^{p}\right\}=\dot{\gamma}\{g, \sigma\}
$$

where $\dot{\gamma}=$ plastic consistency parameter. This multiplier is subject to the following Kuhn-Tucker-Karush conditions: $\dot{\gamma} \geq 0, f \leq 0$, and $\dot{\gamma} f=0$ (i.e., a stress state can only lie on, or within, the perfectly plastic yield envelope).

\section{Hydrostatic Stress versus Principal Stress Ratio Relationship}

In the following sections, it is assumed that once the material point has reached yield, the intermediate principal stress is in the out-ofplane direction. The validity of this assumption is subsequently examined. Combining this assumption with the direction of plastic flow from Eq. (6), the following relationship between the principal values of stress and elastic strain is obtained

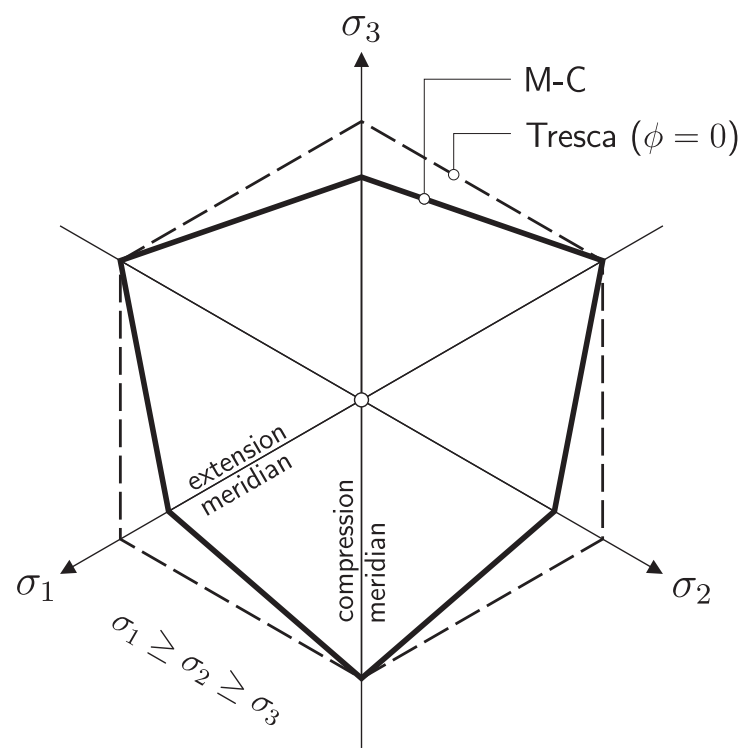

(a)

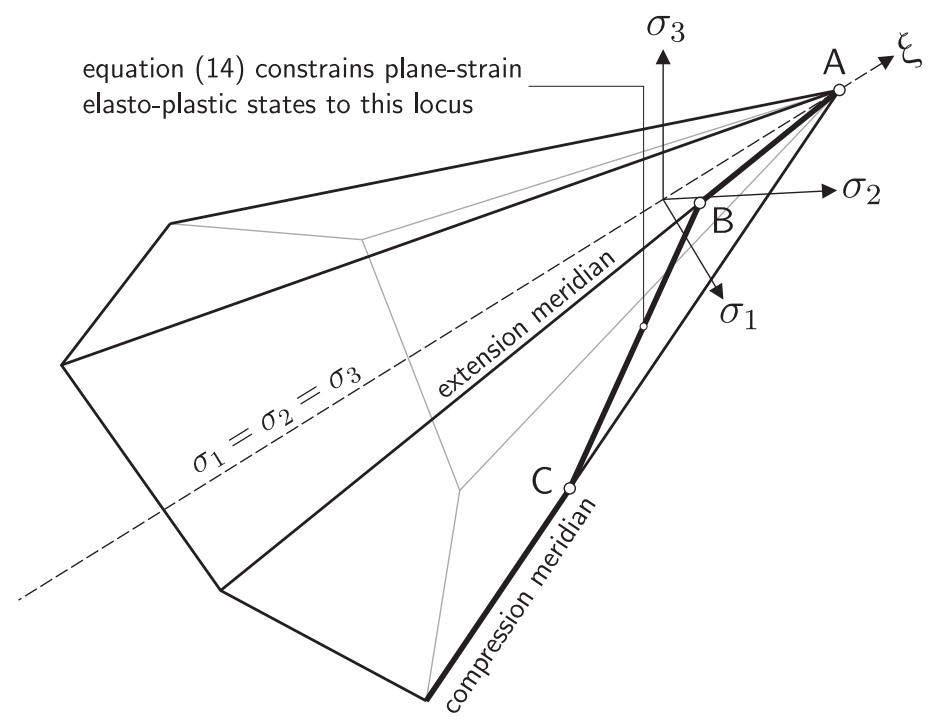

(b)

Fig. 1. Mohr-Coulomb yield surface: (a) deviatoric section (including the Tresca yield criterion); (b) principal stress space including the plane-strain stress relationship [Eq. (14)] 


$$
\{\sigma\}=\left[D^{e}\right]\left\{\begin{array}{lll}
\varepsilon_{1}^{e} & 0 & \varepsilon_{3}^{e}
\end{array}\right\}^{T}
$$

Inverting the elastic stiffness matrix and using the plane-strain condition in Eq. (8), the following relationship between the principal stresses is obtained

$$
\sigma_{2}=\nu\left(\sigma_{1}+\sigma_{3}\right)
$$

Using the definition of $\xi$ and Eq. (9), the intermediate principal stress can be expressed as

$$
\sigma_{2}=\frac{\sqrt{3} \xi \nu}{(1+\nu)}
$$

Given Eq. (5), the minor principal stress may now be written as

$$
\sigma_{3}=k \sigma_{1}-\sigma_{c}
$$

From Eqs. (10) and (11) and $\xi$, the major principal stress is obtained as

$$
\sigma_{1}=\frac{\sigma_{c}+\sqrt{3} \xi /(1+\nu)}{(1+k)}
$$

Eqs. (10)-(12) show that the values of $\sigma_{1}, \sigma_{2}$, and $\sigma_{3}$ are each determined by $\xi$. The locus traced by these equations is shown on the M-C yield surface in the principal stress space for $\phi=\pi / 9, \nu=0.2$, and $c=100 \mathrm{kPa}$ in Fig. 1(b). Substituting Eqs. (10)-(12) into Eq. (1), the principal stress ratio becomes

$$
b=\frac{\sqrt{3} \xi[1-\nu(1+k)]+\sigma_{c}(1+\nu)}{(1-k) \sqrt{3} \xi+2 \sigma_{c}(1+\nu)}
$$

or, alternatively

$$
\xi=\frac{\sigma_{c}(1+\nu)(1-2 b)}{\sqrt{3}[b(1-k)+\nu(1+k)-1]}
$$

Eq. (14) provides an injective function between $b$ and $\xi$.

\section{Compression and Extension Meridians}

In Eq. (13), $b$ attains a value of zero at the following hydrostatic stress:

$$
\xi_{b=0}=\frac{\sigma_{c}(1+\nu)}{\sqrt{3}[\nu(1+k)-1]}
$$

that is, at Point $\mathrm{C}$ in Fig. 1(b). For hydrostatic stresses less than this value, Eqs. (13) and (14) are no longer valid, because the stress point is situated on the compression meridian $\left(\sigma_{1}=\sigma_{2}>\sigma_{3}\right)$. For Poisson's ratio $\nu \geq 1 /(1+k)$, the stress state will never reach the compression meridian, and instead a limit is imposed on the minimum attainable principal stress ratio given by

$$
\lim _{\xi \rightarrow-\infty} b=\frac{1-\nu(1+k)}{1-k} \quad \nu \in[1 /(1+k), 0.5]
$$

The hydrostatic stress associated with $b=1$ is given by

$$
\xi_{b=1}=\frac{\sigma_{c}(1+\nu)}{\sqrt{3}[k-\nu(1+k)]}
$$

shown by Point B in Fig. 1(b). The stress will be located on the extension meridian $\left(\sigma_{3}=\sigma_{2}<\sigma_{1}\right)$ for hydrostatic pressures $\xi_{b=1} \leq \xi \leq \xi_{c}$, where $\xi_{c}=\sqrt{3} c \cot (\phi)$ identifies where the yield surface intersects the hydrostatic axis [Point A in Fig. 1(b)].

To investigate the limits further, the M-C constitutive model was subjected to one-dimensional straining until reaching yield. Young's modulus of $E=10 \mathrm{GPa}$ and Poisson's ratio of $\nu=0.3$ were used for the material's elastic properties. In this simple illustration, the M-C model had a friction angle of $\phi=\pi / 9\left(20^{\circ}\right)$ and an apparent cohesion of $c=100 \mathrm{kPa}$. Under compression, the constitutive model reached yield at the following normalized stress state:

$$
\frac{\{\sigma\}}{c}=\left\{\begin{array}{lll}
-9.724 & -9.724 & -22.690
\end{array}\right\}^{T}
$$

with a normalized hydrostatic pressure of $(\xi / c)=-24.33$. This state agrees with the compressive theoretical limit provided by Eq. (15). Under extension, the stress path reaches yield at a normalized hydrostatic pressure of $(\xi / c)=1.90$, again agreeing with the theoretical limit when $b=1$, given by Eq. (17). Therefore, the limits provided by Eq. (15), for $b=0$ on the compression meridian, and Eq. (17), when $b=1$ on the extension meridian, define the intersection of the stress path with the M-C yield surface for this uniaxial strain case.

\section{Special Case of Tresca (Frictionless) Yielding with Associated Flow}

In the limiting case where $\phi=0$ (i.e., Tresca plasticity), the yield criterion Eq. (5) and the direction of associated plastic flow Eq. (6) become

$$
f=\sigma_{1}-\sigma_{3}-2 c=0 \quad \text { and } \quad\left\{g_{\sigma}\right\}=\left\{\begin{array}{lll}
1 & 0 & -1
\end{array}\right\}^{T}
$$

because $k(\phi=0)=1$. Following the same steps as the M-C case, the principal stresses are obtained as

$\sigma_{1}=\frac{\sqrt{3} \xi}{2(1+\nu)}+c, \quad \sigma_{2}=\frac{\sqrt{3} \xi \nu}{(1+\nu)}, \quad$ and $\quad \sigma_{3}=\sigma_{1}-2 c$

The principal stress ratio then becomes

$$
b=\frac{\sqrt{3}(1-2 \nu)}{4 c(1+\nu)} \xi+\frac{1}{2}=a_{v} \xi+\frac{1}{2} \quad \text { or } \quad \xi=\frac{b-1 / 2}{a_{v}}
$$

where the definition of $a_{v}$ is self-evident. Thus, for the plane-strain analysis using the Tresca yield criterion, a linear relationship exists between $\xi$ and $b$. Similar to the M-C relationship, the limits on Eq. (20) are obtained as

$$
\xi_{b=0}=\frac{2 c(1+\nu)}{\sqrt{3}(2 \nu-1)} \quad \text { and } \quad \xi_{b=1}=\frac{2 c(1+\nu)}{\sqrt{3}(1-2 \nu)}
$$

with $\xi_{b=0}=-\xi_{b=1}$. Beyond these limits, the stress state will be located on the compression $\left(\xi \leq \xi_{b=0}\right)$ or extension meridian $\left(\xi \geq \xi_{b=1}\right)$.

\section{Yield Condition at the Corners}

When material states are on the compression or extension meridians, the direction of plastic flow is no longer uniquely defined. However, given a total strain increment, the plastic strain increment (and therefore the elastic strain and stress increments) can be obtained using the method proposed by Koiter (1953). The nonuniqueness of 
the plastic strain direction can lead to inelastic deformation in the out-of-plane direction.

For the case when $\varepsilon_{2}^{e} \neq 0$, the principal Cauchy stresses are given by

$$
\{\sigma\}=\left[D^{e}\right]\left\{\begin{array}{lll}
\varepsilon_{1}^{e} & \varepsilon_{2}^{e} & \varepsilon_{3}^{e}
\end{array}\right\}^{T}
$$

Subtracting the intermediate elastic strain from each component of the principal strain vector and adding the equivalent hydrostatic pressure, $\sigma^{e}$, to the right side of Eq. (22), gives rise to the following relationship

$$
\{\sigma\}=\left[D^{e}\right]\left\{\left(\varepsilon_{1}^{e}-\varepsilon_{2}^{e}\right) \quad 0 \quad\left(\varepsilon_{3}^{e}-\varepsilon_{2}^{e}\right)\right\}^{T}+\sigma^{e}\{1\}
$$

where $\sigma^{e}=\varepsilon_{2}^{e} E /(1-2 \nu)$ and $\{1\}$ indicate a three-component vector populated with ones. Following the same procedure for deriving Eq. (13), the principal stresses can be expressed as

$$
\begin{aligned}
\sigma_{1} & =\frac{\sqrt{3} \xi-(1-2 \nu) \sigma^{e}+(1+\nu) \sigma_{c}}{(1+k)(1+\nu)} \\
\sigma_{2} & =\frac{\sqrt{3} \xi \nu+(1-2 \nu) \sigma^{e}}{(1+\nu)}, \text { and } \sigma_{3}=k \sigma_{1}-\sigma_{c}
\end{aligned}
$$

Substituting Eq. (24) into Eq. (1), the following relationship for the principal stress ratio is obtained

$$
b=\frac{\sqrt{3} \xi[1-\nu(1+k)]-(1-2 \nu)(2+k) \sigma^{e}+\sigma_{c}(1+\nu)}{\left[\sqrt{3} \xi-(1-2 \nu) \sigma^{e}\right](1-k)+2 \sigma_{c}(1+\nu)}
$$

When $\varepsilon_{2}^{e}=0$, Eq. (25) reduces to Eq. (13). Eq. (25) is bounded between the following levels of hydrostatic stress

$$
\begin{aligned}
\xi_{b=0} & =\frac{\sigma_{c}(1+\nu)-(1-2 \nu)(2+k) \sigma^{e}}{\sqrt{3}[\nu(1+k)-1]} \text { and } \\
\xi_{b=1} & =\frac{\sigma_{c}(1+\nu)+(1-2 \nu) \sigma^{e}}{\sqrt{3}[k-\nu(1+k)]}
\end{aligned}
$$

\section{Examination of the Orientation of $\sigma_{2}$}

Before presenting the results for plane-strain M-C elastoplasticity, the authors examine the validity of the assumption that the intermediate principal stress, $\sigma_{2}$, is the out-of-plane stress, $\sigma_{z}$. The first condition that should be considered is when the principal elastic strains in the in-plane directions are equal. In this case, the stress in the out-of-plane direction $\left(\sigma_{z z}\right)$ is either the major $\left(\sigma_{1}\right)$ or the minor $\left(\sigma_{3}\right)$ principal stress (depending on the sign of $\left.\varepsilon_{2}^{e}\right)$. The resultant stress state will be located on the compression $\left(b=0\right.$, for positive $\left.\varepsilon_{2}^{e}\right)$ or extension $\left(b=1\right.$, for negative $\left.\varepsilon_{2}^{e}\right)$ meridians with hydrostatic stresses less than Eq. (15) or greater than Eq. (17), respectively.

A more interesting case to consider is an unstressed single material point subjected to a stress increment of $\Delta \sigma_{x}=-200 \mathrm{kPa}$, followed by a strain increment of $\Delta \varepsilon_{y}=-1 \times 10^{-3}$. In this illustrative example, the material is modeled by a Young's modulus of $100 \mathrm{MPa}$, Poisson's ratio of 0.3 , friction angle and dilation angle of $\pi / 9$, and an apparent cohesion of $100 \mathrm{kPa}$. The principal stress variation with $\varepsilon_{y}$ and normalized hydrostatic stress $(\xi / c)$ is shown in Figs. 2(a and b), respectively.

Application of $\Delta \sigma_{x}$ causes the stress to move from States 1 to 2 (Fig. 2) with $\sigma_{z}=\sigma_{2}$. From State 2, the stress in the $x$-direction remains constant, during which a strain in the $y$-direction is applied.
At Stage I, the relative proportions of the principal stresses change such that $\sigma_{z}$ is no longer the intermediate principal stress. Between Stage I and Stage III, $\sigma_{z}$ is greater than both $\sigma_{x}$ and $\sigma_{y}$. Along this path, $\sigma_{y}$ is initially the intermediate principal stress. Between Stages II and III, $\sigma_{x}$ is the intermediate principal stress. The ordering changes again at Stage III, where $\sigma_{z}$ becomes the intermediate principal stress. Thereafter, $\sigma_{x}>\sigma_{z}>\sigma_{y}$. The material yields at State 3, with the stresses remaining constant under continuous deformation. The direction of the principal stresses is shown schematically by the cuboids at the top of Fig. 2, where the dashed gray and the solid unshaded cuboids show the original and final deformed shapes, respectively.

This example shows that even for simple linear isotropic elasticity, the direction of the intermediate principal stress in plane-strain analyses is not necessarily directed out of the plane. However, in this example, following yielding, $\sigma_{2}$ is the out-of-plane stress. In the examples which follow, the intermediate principal stresses is the outof-plane stresses at the point of yielding.

\section{Finite-Element Simulations}

\section{Two-Element Simulation}

A simple finite-element simulation using just two four-noded fullyintegrated quadrilateral elements is now considered (Fig. 3). Two upper surface nodes were subjected to vertical displacements of $v=-0.01 \mathrm{~m}$ in 100 equal increments. A Young's modulus of $E=100 \mathrm{MPa}$ and Poisson's ratio of $v=0.3$ were used for the material's elastic properties. As in the previous example, the associated flow M-C model had a friction angle of $\pi / 9$ and a cohesion of $100 \mathrm{kPa}$.

Fig. 3 shows the $\xi / c$ versus $b$ paths for the integration points that underwent elastoplastic deformation during the analysis (i.e., seven out of a total of eight integration points). The initial states are identified by the white symbols, and the states corresponding to a displacement of $10 \mathrm{~mm}$ are shown by the gray shaded symbols. On commencing inelastic straining, the Gauss point $\xi$ versus $b$ paths reach the analytical solution provided by Eq. (14), as shown by the thick light gray line in Fig. 3. Under increasing deformation, the stress states continue to move along that locus.

To highlight the differences between the true M-C yield surface and $\mathrm{M}-\mathrm{C}$ formulations where the local curvature is introduced near the compression and extensions meridians, this simple two-element simulation was analyzed using the $\mathrm{C}^{2}$ continuous M-C surface of Abbo et al. (2011). Before presenting the numerical results, the following disadvantages associated with rounding corners are noted:

- Implicit stress integration (e.g., backward Euler) of a smoothed $\mathrm{M}-\mathrm{C}$ yield surface will generally require multiple iterations to converge. Thus, the rounded version of the M-C is computationally more expensive in terms of both the material point stress integration and the global solution scheme. There are also potential stability issues when returning near the tensile apex on a smoothed yield surface. This is unlike the true M-C envelope, which will always return in one step.

- Rounding corners introduces errors into the stress-integration procedure, whereas the true $\mathrm{M}-\mathrm{C}$ envelope with sharp corners gives an exact stress-integration solution (provided that the corners are dealt with appropriately). Introducing rounding can prohibit the convergence toward established analytical solutions (e.g., Prandtl solution).

Introducing local curvature destroys the unique relationship between hydrostatic stress and the principal stress ratio, as shown in Fig. 4. The smoothed M-C model of Abbo et al. (2011) requires a transition Lode angle, $\theta_{t}$, where the M-C yield surface is smoothed for $|\theta|>\theta_{t}$. Here, $\theta_{t}$ was set to $\pi / 9\left(20^{\circ}\right)$, corresponding to 


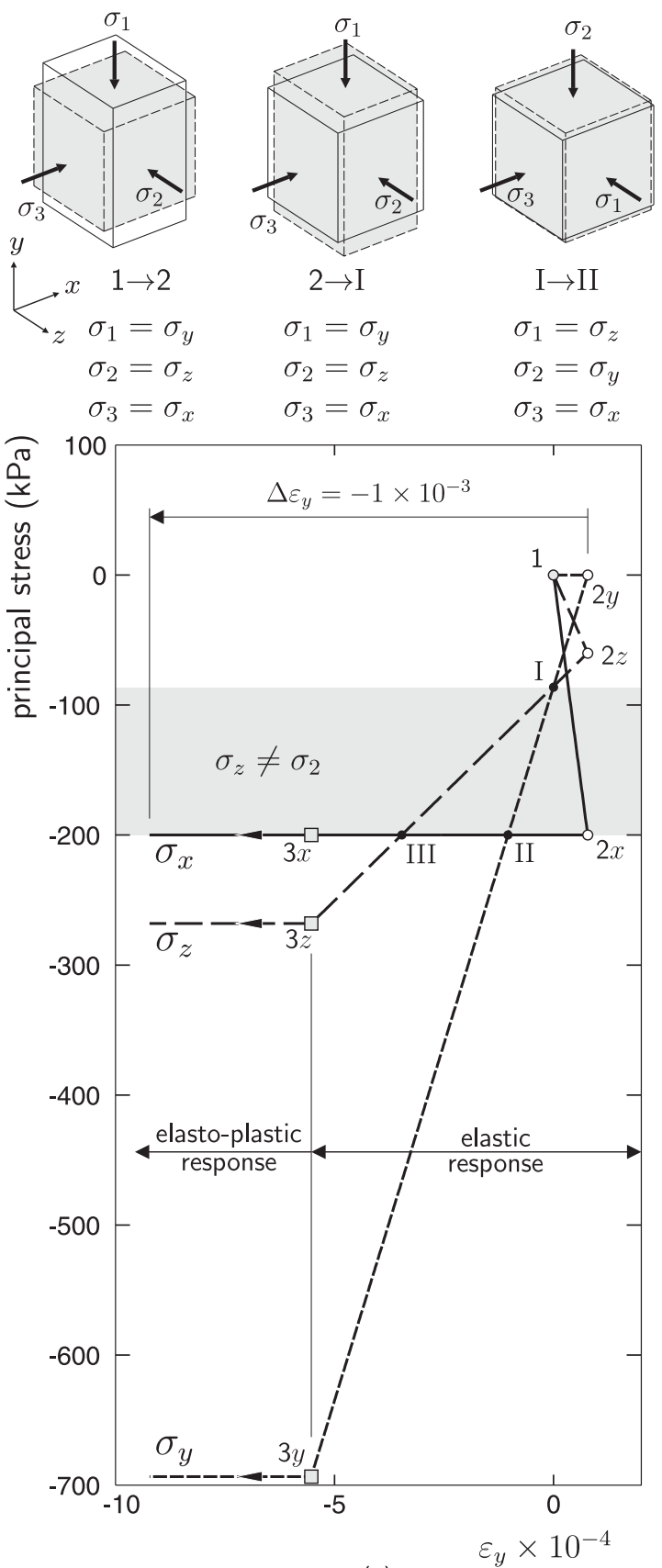

(a)
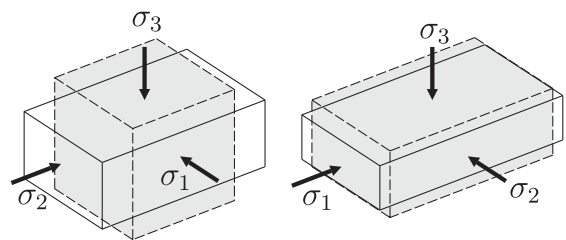

II $\rightarrow$ III

III $\rightarrow$

$\sigma_{1}=\sigma_{z}$

$\sigma_{2}=\sigma_{x}$

$\sigma_{1}=\sigma_{x}$

$\sigma_{3}=\sigma_{y}$

$\sigma_{2}=\sigma_{z}$

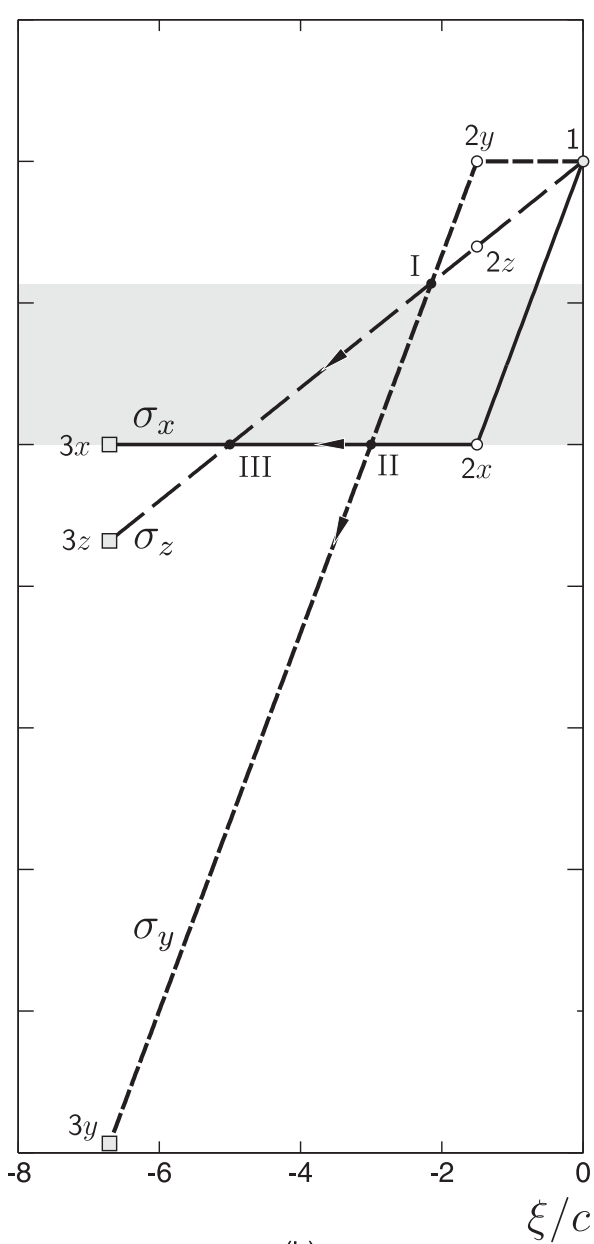

(b)

Fig. 2. Material point analysis: principal stresses against (a) vertical strain and (b) normalized hydrostatic stress, $\xi / c$

transition principal stress ratios, $b_{t}$, of 0.185 and 0.815 . Once a stress state moves into the rounded region in the vicinity of the compression or extension meridians, the numerical $\xi / c$ versus $b$ paths disagree with the analytical solution [Eq. (14)].

As previously mentioned, if appropriately constructed, an implicit stress-integration routine for the true $\mathrm{M}-\mathrm{C}$ envelope will always return in a single step. However, to achieve this, simple geometric rules must be formulated to identify the appropriate return position based on the trial stress state (Clausen et al. 2006). By operating in principal stress space, it is possible to identify which of the following return locations applies: either on the planar surface or at the intersection of two or more surfaces. In the latter case, the intersection may occur on the compression meridian, on the extension meridian, or at the tensile apex. This process circumvents the instability issues potentially associated with iterative approaches.
The cost of the numerical analysis is now considered. The model with local curvature in the yield surface required 250 global iterations, whereas the true M-C yield surface only required 181. Also, the smoothed M-C model required multiple material point iterations to obtain convergence in the stress-integration routine during each of these global iterations. The combination of these two factors resulted in a $255 \%$ increase in the overall run time when using the smoothed $\mathrm{M}-\mathrm{C}$ approximation.

\section{Footing Analysis}

This section presents the numerical analysis of a 1-m-wide rigid strip footing bearing onto a weightless soil using the M-C model. Because of symmetry, only one-half of the 5 by $10 \mathrm{~m}$ domain was discretized using 135 eight-noded quadrilateral elements with reduced 


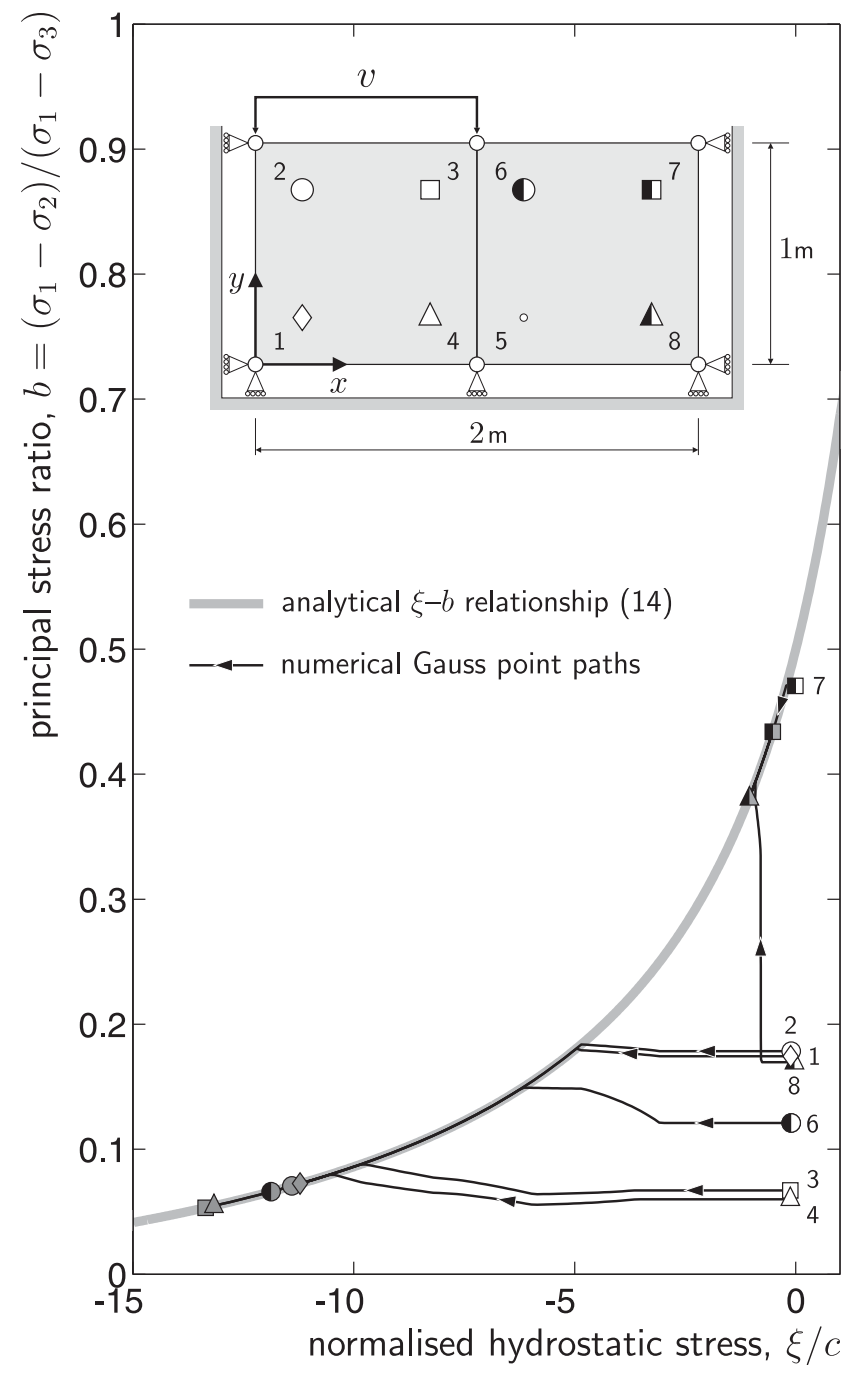

Fig. 3. $\xi / c$ versus $b$ paths for the two-element simulation for $v \leq 10 \mathrm{~mm}$ using the true Mohr-Coulomb surface

four-point Gaussian quadrature (as shown to the right of Fig. 5, where the lower inset figure shows the global discretization and the upper figure shows the mesh refinement detail around the footing). This is the same mesh adopted by de Souza Neto et al. (2008) and later used by Coombs et al. (2010) for the small-strain analysis of frictional cone models. A Young's modulus of $E=100 \mathrm{MPa}$ and Poisson's ratio of $\nu=0.3$ were used for the material's elastic properties. The $\mathrm{M}-\mathrm{C}$ model again had a friction angle of $\pi / 9$ and a cohesion of $100 \mathrm{kPa}$. The analysis was performed using both associated $\left(\phi_{g}=\pi / 9\right)$ and nonassociated $\left(\phi_{g}=\pi / 18\right)$ plastic flow rules. The rigid strip footing was subject to a uniform vertical displacement of $100 \mathrm{~mm}$ in 100 equal steps. The normalized pressure versus displacement response is shown in Fig. 5.

The theoretical limit pressure for the M-C model, as given by the Prandtl (and Reissner) solution [see Yu (2006) among others for details] is

$$
p=c\left[\tan ^{2}(\pi / 4+\phi / 2) \exp ^{\pi \tan (\phi)}-1\right] \cot (\phi)
$$

This equation gives the limit pressure for a rigid footing bearing onto a weightless soil for the case of zero surface surcharge. For a friction angle of $\pi / 9$, the normalized theoretical limit pressure is $(p / c)=14.84$. Both the associated and nonassociated flow

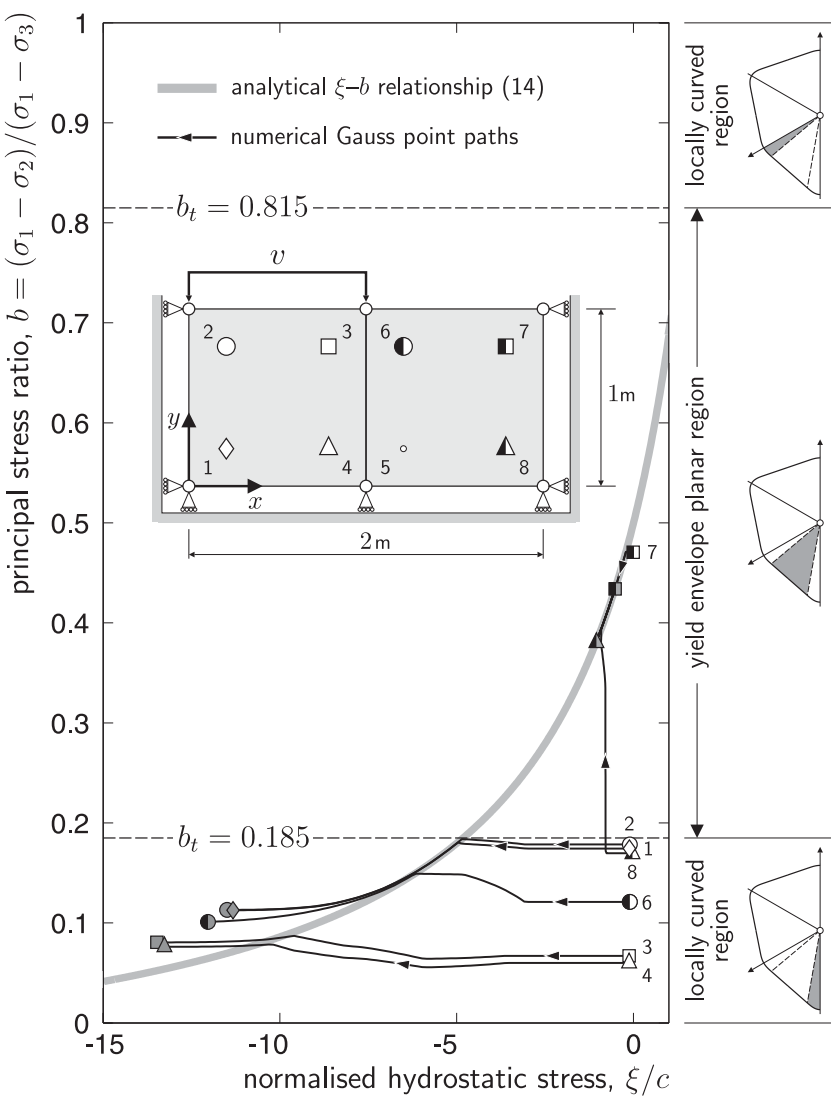

Fig. 4. $\xi / c$ versus $b$ paths for the two-element simulation for $v \leq 10 \mathrm{~mm}$ using the $\mathrm{C}^{2}$ continuous smoothed Mohr-Coulomb surface of Abbo et al. (2011)

simulations agree rather well with this theoretical limit load, having errors of just 0.46 and $0.74 \%$, respectively.

Fig. 6(a) shows the principal stress ratio versus normalized hydrostatic stress for the nonassociated finite-element simulation at the end of the analysis (circular discrete points), the path taken to reach that state (fine gray lines), and the analytical $\xi$ versus $b$ solution (thick solid black line). Two Gauss point stress paths have been identified by fine black lines, starting at the gray squares ( $\mathrm{G}$ and $\mathrm{H})$ and finishing at the white circular symbols on the analytical solution locus. The final stress states and the elastoplastic stress paths agree with the analytical solution, verifying the unique relationship between $\xi$ and $b$ provided by Eq. (14).

To highlight the special nature of the M-C constitutive formulation, the elastoplastic $\xi$ versus $b$ points (at the end of the finiteelement analysis) for a isotropic linear elastic-perfectly plastic Drucker-Prager (D-P) model (Drucker and Prager 1952) have been plotted in Fig. 6(b). The nonassociated flow D-P model used here had the same elastic properties as the M-C model. The conical D-P model has a circular deviatoric section with the yield surface centered on the hydrostatic axis. This yield envelope provides a simplified smooth approximation to the M-C yield criterion. In this analysis, the D-P cone was chosen to coincide with the M-C surface on the compression meridian. The square symbols in Fig. 6(b) show that, unlike the M-C model, there is no unique $\xi$ versus $b$ plane-strain relationship for the D-P model.

The major difference between the M-C and the D-P models is that, for the D-P model, the yield surface

$$
f=\rho+\alpha\left(\xi-\xi_{c}\right)=0
$$



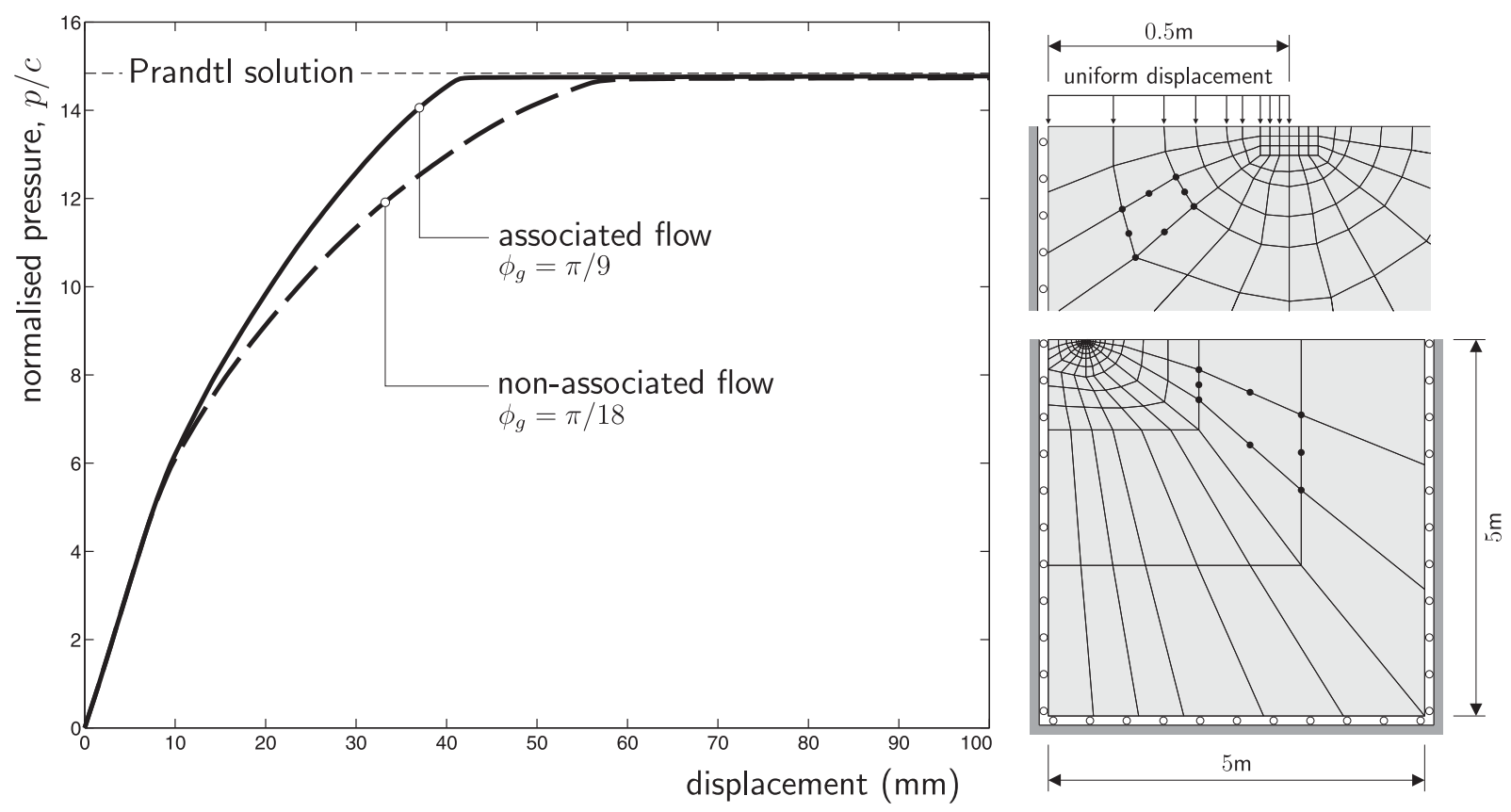

Fig. 5. Rigid strip footing: $p / c$ versus the displacement response and the finite-element discretization

has a dependence on the intermediate principal stress; thus, the associated direction of the plastic flow contains a component in the intermediate principal strain direction. The combination of these two features means that it is not possible to write a unique plane-strain relationship between the hydrostatic stress and the principal stress ratio for the D-P model. In Eq. (28), $\alpha$ is the opening angle of the D-P cone, here set to $\alpha=\tan (\phi), \rho=\sqrt{2 J_{2}}$ is a scalar measure of the deviatoric stress, and $\xi_{c}=\sqrt{3} c \cot (\phi)$ identifies where the yield surface intersects the hydrostatic axis.

\section{Finite-Deformation Cylindrical Cavity Expansion}

In this section we present an analysis of the expansion of a cylindrical soil cavity under internal pressure. Although this can be analyzed as a one-dimensional axisymmetric problem, the twodimensional plane-strain finite-deformation finite-element code is used to make comparisons with an analytical solution and provide further verification of the $\xi-b$ relationship. Only a $3^{\circ}$ segment of the structure (with an internal radius of $1 \mathrm{~m}$ and a fixed outer boundary of radius $2 \mathrm{~km}$ ) was discretized using 50 four-noded plane-strain quadrilateral elements. The size of the elements was progressively increased by a factor 1.12 from the inner to the outer surface. Young's modulus of $E=100 \mathrm{MPa}$ and Poisson's ratio of $\nu=0.2$ were used for the material's elastic properties. The associated flow M-C model had a friction angle of $\pi / 6$ and a cohesion of $100 \mathrm{kPa}$. The internal radius was expanded to $5 \mathrm{~m}$ using 80 equal displacement increments.

Because of this large change in the internal radius, the effects of geometric nonlinearity cannot be ignored. The M-C model described was implemented within a Lagrangian finite-deformation finite-element code. The use of a logarithmic strain-Kirchhoff stress formulation, combined with an exponential map of the plastic flow, allows the incorporation of existing small strain constitutive algorithms without modifying their stress-integration routine. This method is one of the most successful and straightforward ways of accounting for the additional geometric complexities inherent in finite-deformation analyses when implementing large-strain elastoplasticity (Kim et al. 2009). The Kirchhoff stress, $[\tau]$ is defined as

$$
[\tau]=J[\sigma]
$$

where $[\sigma]=$ Cauchy stress, and $J=$ determinant of the deformation gradient. This volume ratio, $J$, is a measure of the change in volume between the current (deformed) configuration and the original reference state. See Coombs and Crouch (2011) and the references contained within for further information on the finite-deformation finite-element formulation.

Fig. 7 shows the normalized internal pressure $(p / c)$ versus expansion ratio $\left(a / a_{0}\right)$ response from the M-C finite-deformation finite-element simulation (solid line), where $a_{0}$ and $a$ are the original and current internal radii, respectively. The numerical results display good agreement with the analytical solution (discrete points) provided by $\mathrm{Yu}$ and Houlsby (1991).

The unique $\xi$ versus $b$ relationship still holds for the finitedeformation analysis provided that the Cauchy hydrostatic stress in Eq. (13) and (14) is replaced by the equivalent Kirchhoff stress measure, namely

$$
\xi_{\tau}=\operatorname{tr}([\tau]) / \sqrt{3}
$$

This is demonstrated in Fig. 8, where the numerical elastoplastic normalized Kirchhoff hydrostatic stress versus principal stress ratio points, at the end of the analysis, have been plotted alongside the analytical relationship (solid line). Additional stress states exist on the compression meridian where $\xi_{\tau} / c<-20$. However, for clarity, the abscissa has been limited to $\xi_{\tau} / c \in[-20,5]$. All of the finiteelement Gauss points lie on the line described by the rational relationship [Eq. (13)].

Ewing and Griffiths (1971) investigated elastoplastic stress concentrations around a notch for an isotropic elastic-perfectly plastic Tresca constitutive model. Their study was based on the plane-strain numerical analysis of Griffiths and Owen (1971). They found that the maximum stress was "attained inside the plastic zone surrounding the notch, not at its edge" (Ewing and Griffiths 1971). This was because of plastic strains, comparable with the in-plane strains, being induced in the out-of-plane direction at material points where the stress state was located on the compression or extension 


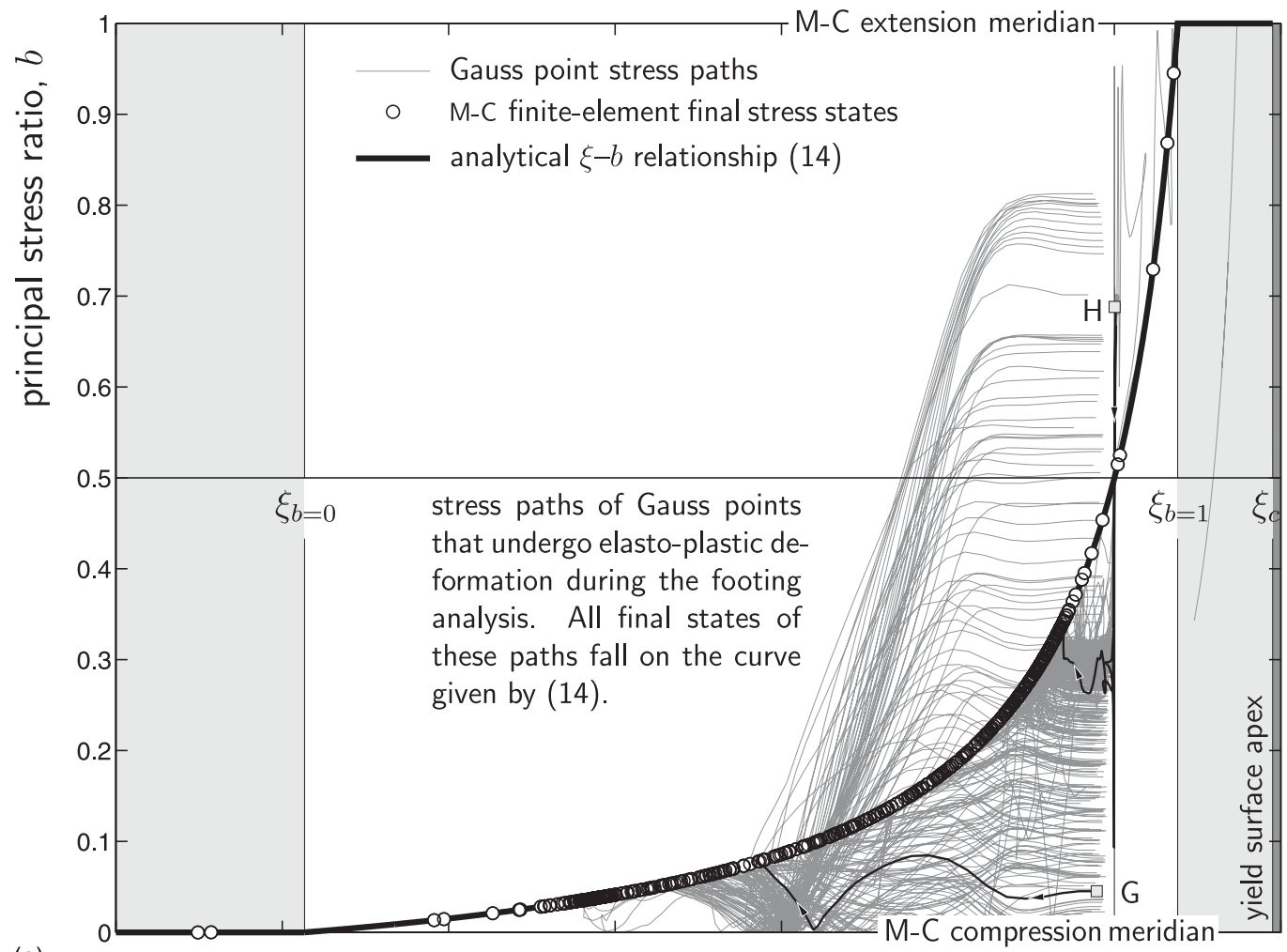

(a)

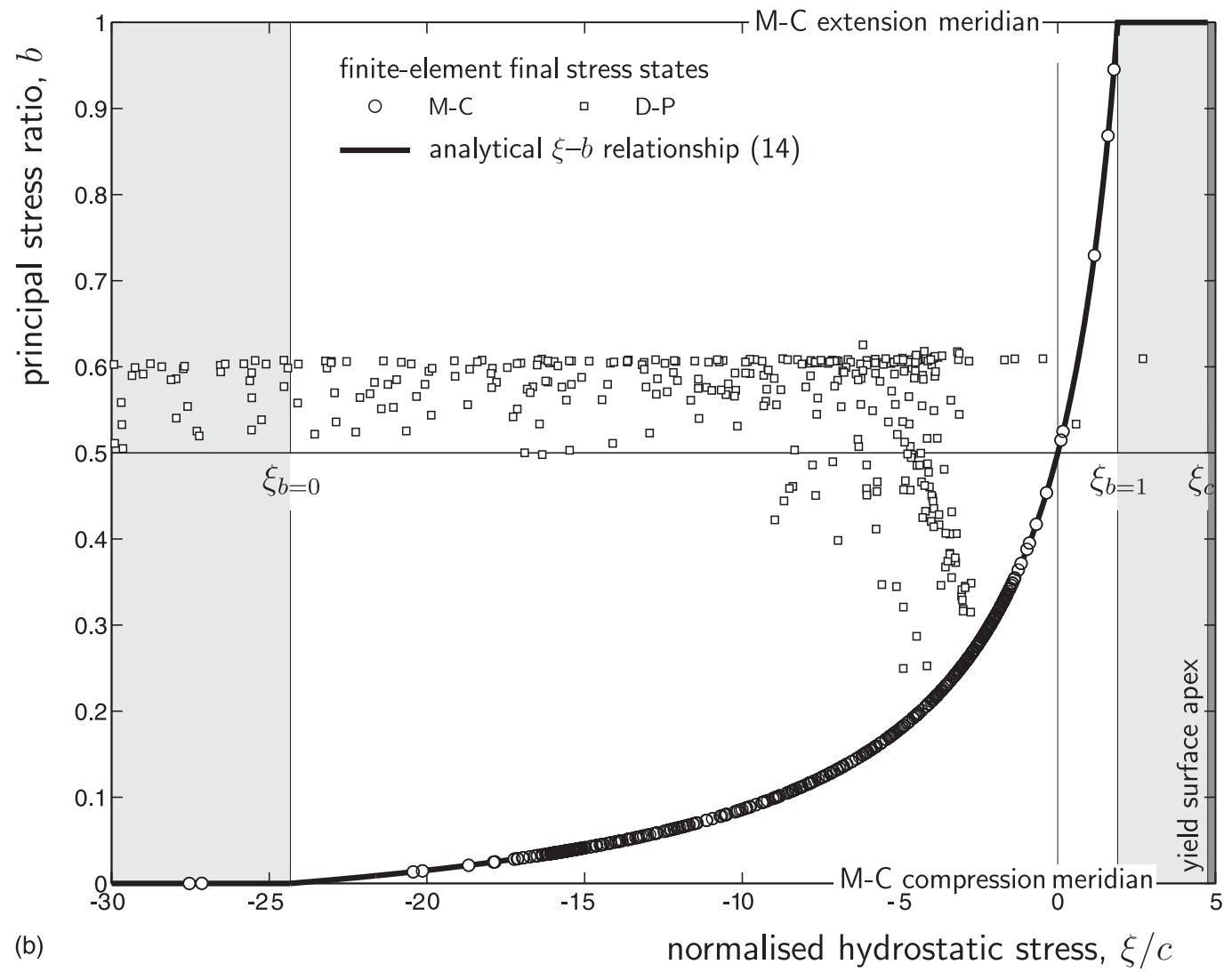

Fig. 6. Rigid strip footing: (a) nonassociated Mohr-Coulomb $\xi / c$ versus $b$ stress path response and (b) stress states at the end of the analysis for the Mohr-Coulomb and Drucker-Prager models 


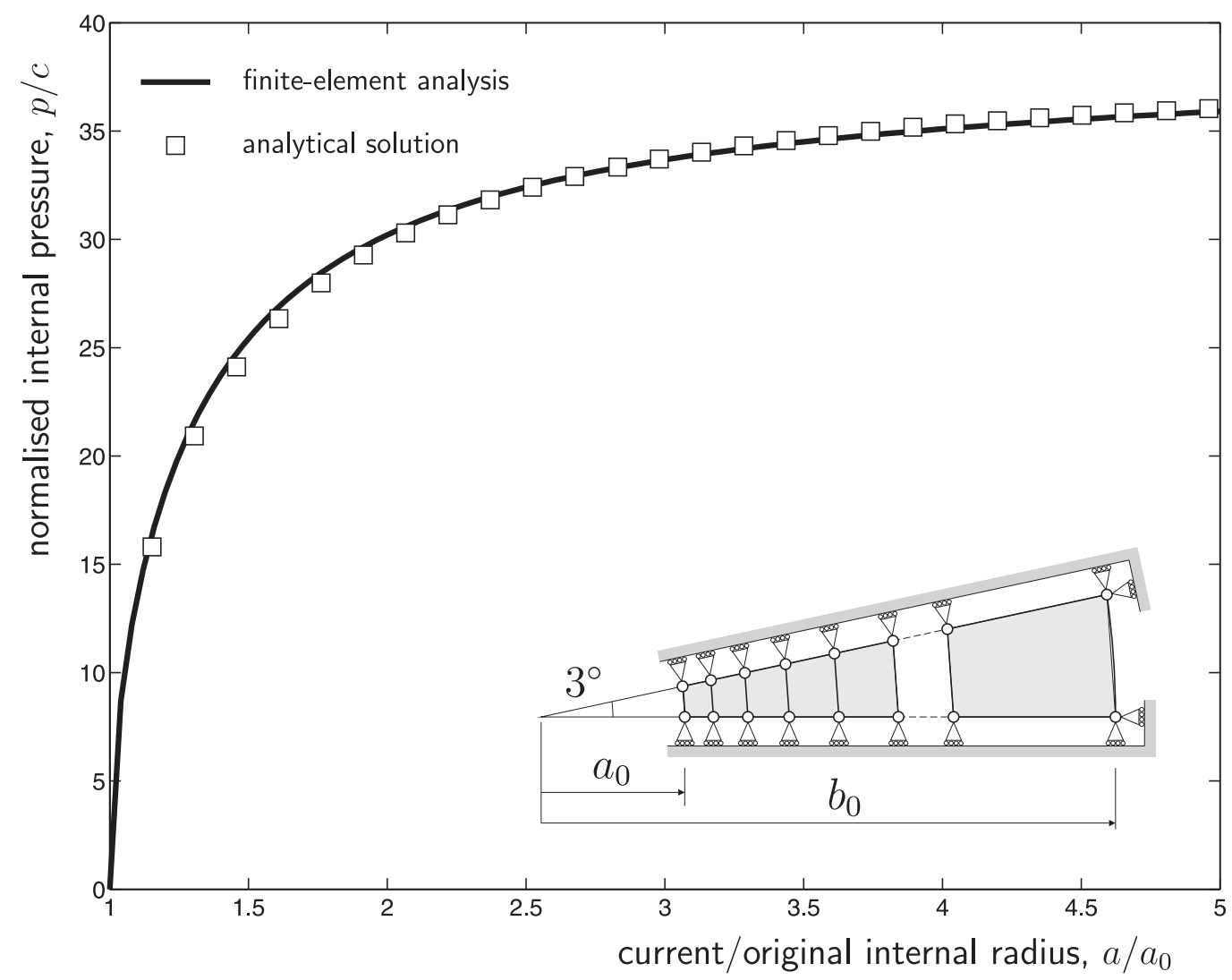

Fig. 7. Cavity expansion: $p / c$ versus $a / a_{0}$

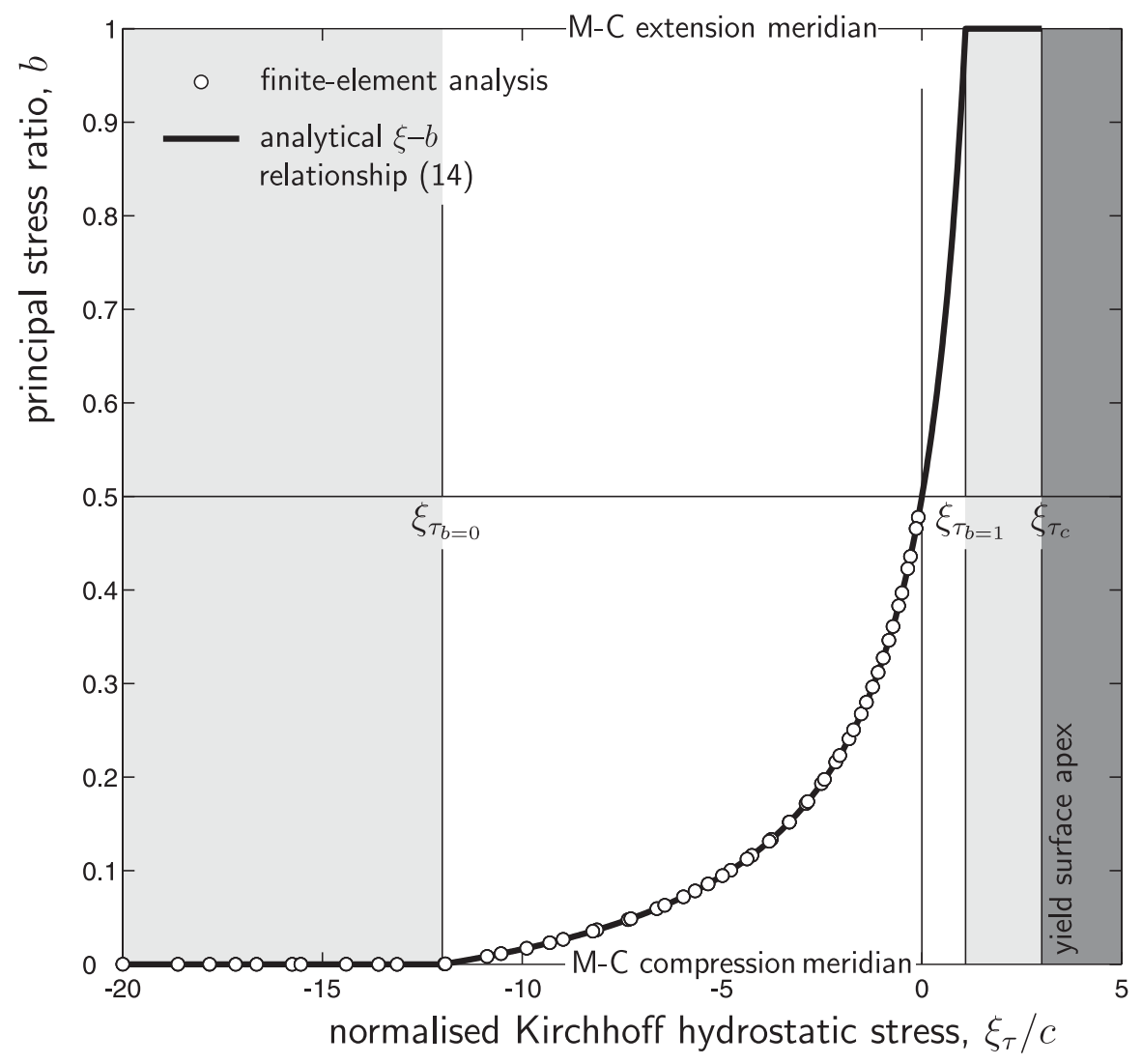

Fig. 8. Cavity expansion: $\xi_{\tau} / c$ versus $b$ response at the end of the analysis 


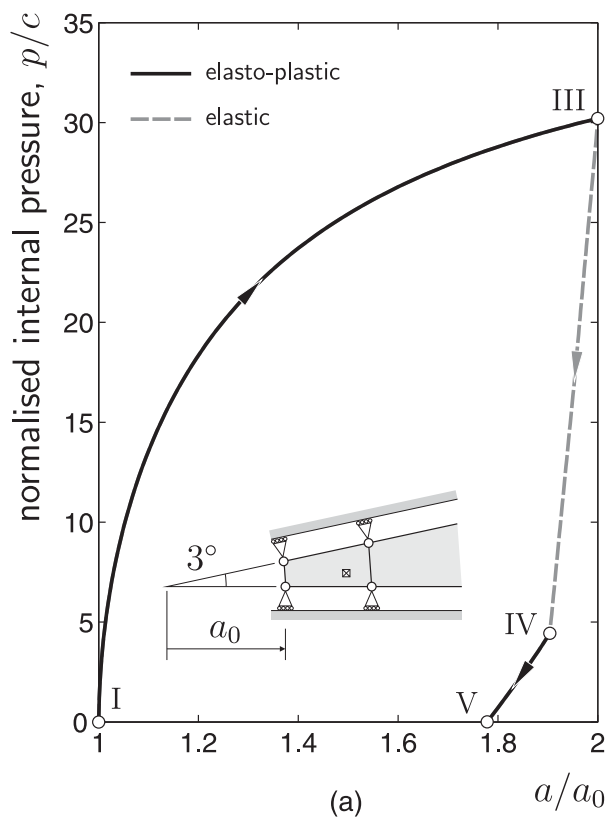

(a)

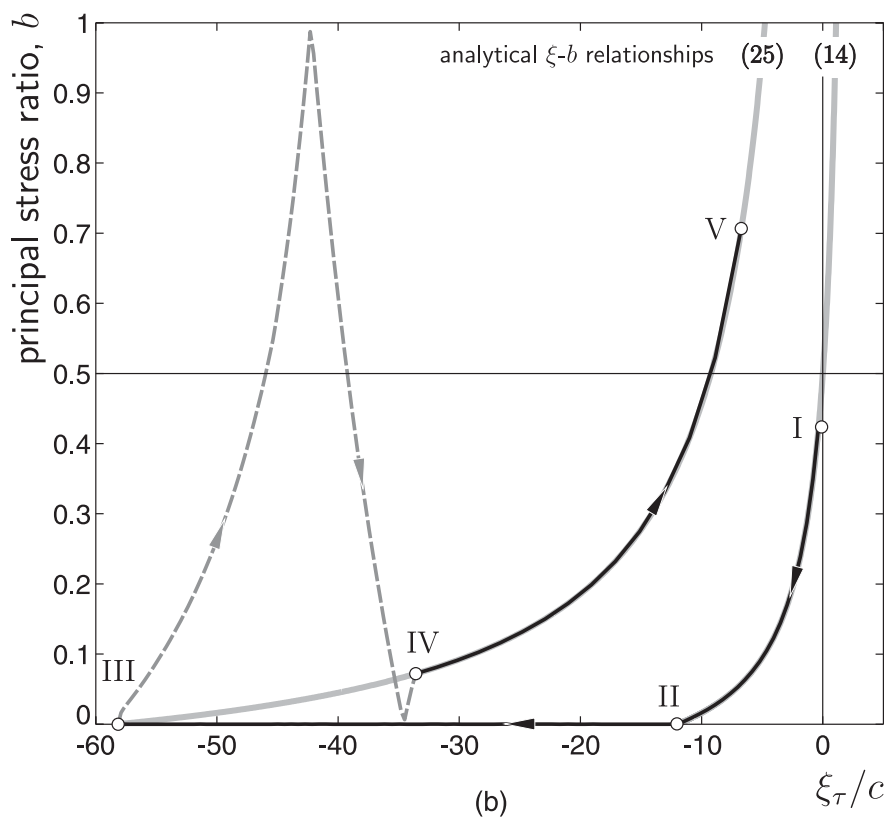

(b)

Fig. 9. Cavity expansion load-unload response: (a) $p / c$ versus $a / a_{0}$; (b) $\xi_{\tau} / c$ versus $b$ path

meridians. Prompted by these findings, Fig. 9 presents the normalized pressure versus expansion response of the cylindrical cavity following loading to an internal pressure of approximately $3 \mathrm{MPa}$ (corresponding to an expansion of $a / a_{0}=2$ ) and then unloading. The load-controlled numerical analysis was conducted using 100 steps in both the loading and unloading phases. The same material parameters used in the previous cavity expansion simulation were used in this analysis. The elastic and elastoplastic sections of the structural response are identified by the dashed gray and solid black lines, respectively. The analysis starts at I and is loaded to III. The structure unloads elastically between III and IV until the reoccurrence of elastoplastic deformation at IV, which continues to $\mathrm{V}$.

The normalized hydrostatic stress versus the principal stress ratio path of a Gauss point, located at an initial radial coordinate of $1.626 \mathrm{~m}$, is shown in Fig. 9(b). Upon loading, the material point intersects with and moves along the locus described by Eq. (13). At II, the stress state reaches the compression meridian. On the compression meridian, the direction of plastic flow is no longer uniquely defined. However, the constitutive model's plastic strain increment (when subjected to a total strain increment) can be obtained using the method proposed by Koiter (1953). The nonuniqueness of the plastic strain direction leads to inelastic deformation in the out-of-plane direction when loading between II and III. That is, the assumption that $\varepsilon_{2}^{p}=0$ is invalidated, and instead the condition $\varepsilon_{2}^{p}=-\varepsilon_{2}^{e}$. The effect of this nonzero out-of-plane elastic strain can be seen in Fig. 9(b). Upon unloading, the stress state moves from III to IV, where it again encounters the yield surface. However, because of the nonzero $\varepsilon_{2}^{e}$, the unloading elastoplastic $\xi / c$ versus $b$ response (between IV and $\mathrm{V}$ ) does not agree with the analytical solution [Eq. (13)]. This solution is restricted to cases where the stress states do not move on to and subsequently away from the corner or apex regions.

Fig. 9(b) shows that the analytical solution when $\varepsilon_{2}^{e} \neq 0$ agrees with the elastoplastic stress path between IV and V. Along this path, the Gauss point had an out-of-plane elastic strain of $\varepsilon_{2}^{e}=-0.0032$. Eq. (25) allows an analytical relationship between $\xi$ and $b$ to be defined for material points that have nonzero elastic strain in the out-of-plane direction. It also supports the findings of Ewing and Griffiths (1971), in that, for a given principal stress ratio, the hydrostatic stress (and hence $\{\sigma\}$ ) can change in magnitude for material points undergoing inelastic straining in the out-of-plane direction.

\section{Conclusions}

This paper has shown that when the out-of-plane stress is the intermediate principal stress, a unique relationship exists between the hydrostatic pressure and the principal stress ratio (or equivalently the Lode angle) for isotropic M-C and Tresca linear elastic-perfectly plastic models in plane-strain analyses. This finding is verified using three numerical simulations, including the analysis of a rigid strip footing bearing onto a weightless soil and the finite-deformation simulation of a cylindrical cavity expansion.

The single-valued function [Eq. (13)] and the extension to the case of $\varepsilon_{2}^{p} \neq 0$ [Eq. (25)] provide new insight into the role of the intermediate principal stress in the $\mathrm{M}-\mathrm{C}$ and Tresca plane-strain analyses.

In this paper, the authors have made use of established procedures for dealing with nonsmooth yield surfaces [e.g., see Clausen et al. (2006, 2007)], which build on the work of Koiter (1953). Some workers have introduced local curvature near the compression and extension meridians, when approximating the $\mathrm{M}-\mathrm{C}$ model, to remove the corners (Abbo et al. 2011). The authors believe that this is quite unnecessary.

\section{References}

Abbo, A., Lyamin, A., Sloan, S., and Hamble, J. (2011). "A C2 continuous approximation to the Mohr-Coulomb yield surface." Int. J. Solids Struct., 48(21), 3001-3010.

Clausen, J., Damkilde, L., and Andersen, L. (2006). "Efficient return algorithms for associated plasticity with multiple yield planes." Int. $J$ Numer. Methods Eng., 66(6), 1036-1059.

Clausen, J., Damkilde, L., and Andersen, L. (2007). "An efficient return lgorithm for non-associated plasticity with linear yield criteria in principal stress space." Comput. Struct., 85(23-24), 1795-1807. 
Coombs, W., and Crouch, R. (2011). "Non-associated Reuleaux plasticity: Analytical stress integration and consistent tangent for finite deformation mechanics." Comput. Methods Appl. Mech. Eng., 200(9-12), 1021-1037.

Coombs, W., Crouch, R., and Augarde, C. (2010). "Reuleaux plasticity: Analytical backward Euler stress integration and consistent tangent." Comput. Methods Appl. Mech. Eng., 199(25-28), 1733-1743.

de Souza Neto, E., Perić, D., and Owen, D. (2008). Computational methods for plasticity: Theory and applications, Wiley, New York.

Drucker, D., and Prager, W. (1952). "Soil mechanics and plastic analysis or limit design." Q. Appl. Math., 10, 157-164.

Ewing, D., and Griffiths, J. (1971). "The applicability of slip-line field theory to contained elastic-plastic flow around a notch." J. Mech. Phys. Solids, 19(6), 389-394.
Griffiths, J., and Owen, D. (1971). "An elastic-plastic stress analysis for a notched bar in plane strain bending." J. Mech. Phys. Solids, 19(6), 419-431.

Kim, D.-N., Montáns, F., and Bathe, K. (2009). "Insight into a model for large strain anisotropic elasto-plasticity." Comput. Mech., 44(5), 651-668.

Koiter, W. (1953). "Stress-strain relations, uniqueness and variational theorems for elastic-plastic materials with a singular yield surface." Q. Appl. Math., 11, 350-354.

Shield, R. (1955). "On Coulomb's law of failure in soils." J. Mech. Phys. Solids, 4(1), 10-16.

Yu, H.-S. (2006). Plasticity and geotechnics, Springer, New York.

Yu, H.-S., and Houlsby, G. (1991). "Finite cavity expansion in dilatant soils: Loading analysis." Geotechnique, 41(2), 173-183. 